\title{
Investigating the Effects of Physical Therapy Timing, Intensity and Duration on Post-Traumatic Joint Contracture in a Rat Elbow Model
}

\author{
A. J. Reiter ${ }^{1}$, R. M. Castile1, H. R. Schott ${ }^{2}$, G. J. Kivitz ${ }^{1}$, A. M. Chamberlain ${ }^{3}$, S. P. Lake ${ }^{1-3}$ \\ 1 Department of Mechanical Engineering and Materials Science, Washington University in St. Louis, \\ St. Louis (MO), U.S.A. \\ 2 Department of Biomedical Engineering, Washington University in St. Louis, St. Louis (MO), U.S.A. \\ 3 Department of Orthopaedic Surgery, Washington University in St. Louis, St. Louis (MO), U.S.A.
}

\section{CORRESPONDING AUTHOR:}

Spencer P. Lake

Department of Mechanical Engineering and Materials Science

Washington University in St. Louis

1 Brookings Drive

St. Louis (MO) 63130-4899 U.S.A.

E-mail: lake.s@wustl.edu

DOI:

10.32098/mltj.03.2021.20

LEVEL OF EVIDENCE: $1 B$

\begin{abstract}
SUMMARY
Background. Post-traumatic joint contracture (PTJC), characterized by loss of motion and permanent stiffness, affects up to $50 \%$ of patients following elbow joint dislocation or fracture. Mechanisms governing successful conservative treatment methods aimed at preventing elbow PTJC and avoiding operative treatments (e.g., physical therapy) are poorly understood. Using a previously established rat model of elbow PTJC, the purpose of this study was to explore the effect of varying timing, intensity and duration of active, functional exercise on joint motion outcomes.

Methods. Following a surgically-induced unilateral elbow dislocation in rats, injured limbs were immobilized in bandages for 42 days followed by free mobilization for 42 additional days producing long-term PTJC. This work summarizes several studies (Phases I-III) that investigated the effects of early versus delayed therapy (timing), free mobilization versus forced treadmill walking (intensity), and limited-time versus unlimited use (duration) on elbow PTJC.

Results. Joint motion outcomes in therapy groups showed no improvements compared to non-treated injured animals when therapy began day 14 post-injury or later regardless of timing, intensity or duration. Improved joint range-of-motion was only achieved when bandages were permanently removed at day 3 post-injury, regardless of whether added treadmill walking was performed.

Conclusions. Early motion is essential to preserving range-of-motion following traumatic elbow injury in a rat model.
\end{abstract}

\section{KEY WORDS}

Elbow; joint contracture; physical therapy; range-of-motion; rat model; treadmill walking.

\section{BACKGROUND}

Post-traumatic joint contracture (PTJC), characterized by loss of motion and permanent stiffness, affects up to $50 \%$ of patients following elbow joint dislocation or fracture $(1,2)$. Not only is the elbow the most commonly dislocated joint in the pediatric population and second most common in adults (3), it is also particularly susceptible to PTJC due to its high degree of joint congruency and constraint $(4,5)$. Conservative treatment protocols to prevent elbow PTJC including physical therapy, bracing, and manual manipulation have varying degrees of success (6-10). In more severe elbow injuries, permanent functional deficits often remain with nearly $12-15 \%$ of patients requiring surgical intervention when standard non-operative treatments are unsuccessful (11). While conservative treatment protocols such as physical therapy are desirable to prevent PTJC and avoid operative treatments, mechanisms governing successful protocols are poorly understood (6). To date, published studies on this topic have been predominantly case series or retrospective analyses, 
so there is little comparative data with which to evaluate different approaches $(6,12-15)$. The lack of randomized control trials makes determination of optimal clinical treatment approaches challenging, leading to subjective and inconsistent physical therapy protocols.

Despite the lack of guidance on specific levels of physical therapy dosage and timing, clinicians generally agree that active, functional exercises along with patient education are key components to a successful rehabilitation program (16). An animal model offers an ideal approach to test and define general therapy guidance focused on active, functional exercises aimed at preventing elbow PTJC. Animal models allow for a repeatable injury pattern, consistent therapy protocol and controlled joint testing. To this end, a previously established rat model of elbow PTJC that shows functional, mechanical and histological changes similar to human patients (17-20) was leveraged to explore the effect of varying timing, intensity and duration of active, functional exercise on joint motion outcomes. This work summarizes several studies (Phases I-III) that investigated the effects of early versus delayed therapy (timing), free mobilization versus forced treadmill walking (intensity), and limited-time versus unlimited use (duration) on elbow PTJC. We hypothesized that (1) a higher intensity therapy of forced treadmill walking as compared to free mobilization would increase joint motion outcomes when physical therapy is delayed (Phases I and II), and (2) a longer duration therapy of unlimited use as compared to limited-time use would increase joint motion outcomes when physical therapy is administered early (Phase III).

\section{MATERIALS AND METHODS}

\section{Injury model}

In this Institutional Animal Care and Use Committee approved study, a clinically-relevant elbow dislocation was surgically-induced in male Long-Evans rats ( $\mathrm{n}=10$, Charles River Laboratories, Wilmington, MA, USA) $(17,18)$. While anesthetized, one elbow of each animal was subjected to a lateral collateral ligament transection and anterior capsulotomy to replicate the soft tissue damage that occurs during a simple elbow dislocation injury in humans (figure 1). Due to surgery complications, one animal was excluded, leaving nine injured animals. Following surgery, the injured forelimbs were immobilized in bandages for 42 days. Bandages were removed and animals were allowed free mobilization (unrestricted cage activity) for an additional 42 days. Age-matched control animals $(n=10)$ received no injury and were allowed free mobilization for the entire 84-day period (figure $2 \mathrm{~A}$ ).
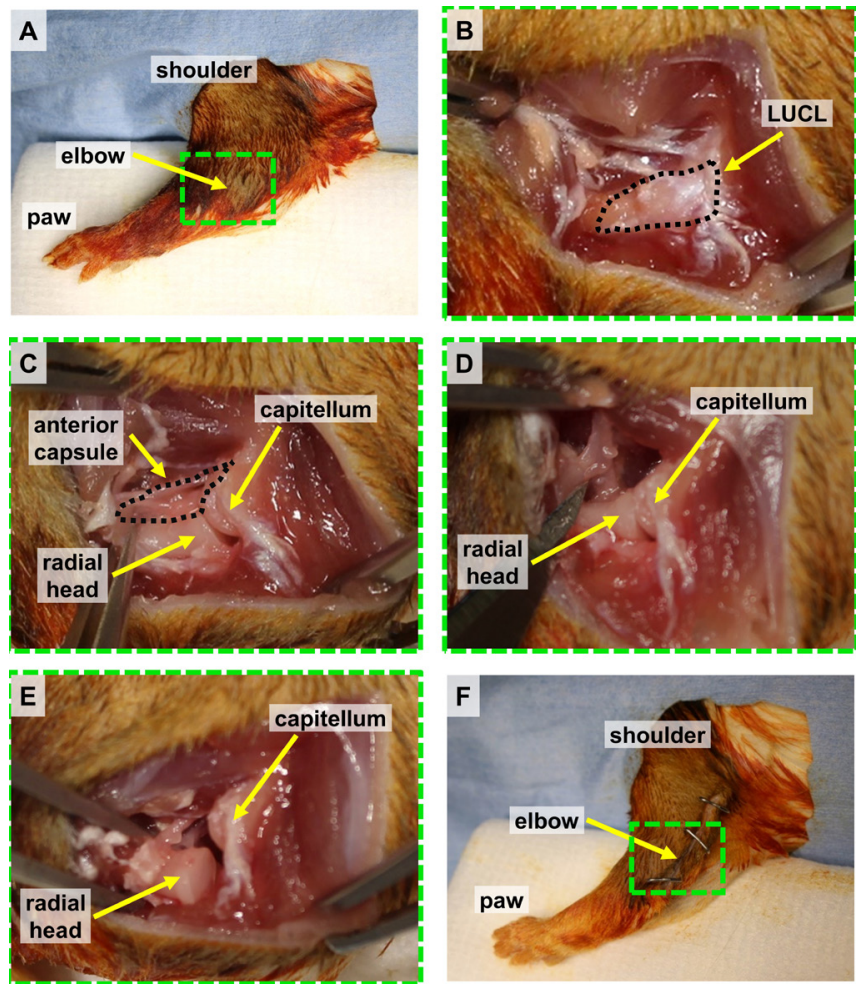

Figure 1. (A) The left forelimb of the animal was prepared for surgery under sterile conditions. (B) Surgical exposure consisted of creating full-thickness skin flaps to the level of forelimb muscle fascia on the lateral side of the elbow. This provided ample visualization of the lateral ulnar collateral ligament (LUCL) and adjacent anterior capsule. (C) The LUCL was transected and the radiocapitellar joint was then visualized. The anterior capsule is evident anterior to the joint space. (D) The capsule (shown here at the tip of the scalpel blade) was divided. (E) Once the LUCL had been divided, the radiocapitellar joint could be subluxated posteriorly, confirming complete transection of the LUCL. (F) The wound was closed in layered fashion with sutures in the muscular layer and staples on the skin.

This is a simulated surgery on a euthanized animal.

\section{Therapy and treadmill protocols}

Experimental groups consisted of three chronologically conducted studies (figure 2, Phases I-III). Physical therapy to the injured elbow was administered by increasing the intensity of forelimb use via forced, flat treadmill walking and/or varying the timing and duration of free mobilization. A commercially available, home fitness treadmill (Horizon Fitness, Cottage Grove, WI, USA) was modified to reduce the speed range suitable for walking rodents (figure 3 ). Briefly, four small magnetics were spaced evenly around the front 
roller (as opposed to the original single magnet) causing the speed sensor to maintain the belt speed at a four-fold reduction from the displayed speed. Custom acrylic boxes with lanes were built and mounted on the treadmill to restrict movement along the direction of belt motion (figure 3).

Phase I investigated if forced treadmill walking would improve joint motion outcomes to a greater extent than free mobilization alone (figure $2 \mathrm{~A}$ ). Following elbow dislocation surgery and 42 days of immobilization, animals $(n=3)$ were allowed free mobilization for 7 days (i.e., study days 42-49). For the remaining 35 days of the free mobilization period (i.e., study days 49-84), animals were subjected to treadmill walking for 60 minutes at $10 \mathrm{~m} / \mathrm{min} 3 \times /$ week. Previous studies subjecting rats to treadmill walking used protocols with up to 60 minutes per session at $12 \mathrm{~m} / \mathrm{min} 6 \mathrm{x} /$ week $(21,22)$. After reviewing results from Phase I, Phase II was developed to begin therapy earlier and alter intensity while maintaining the established 42-day immobilization period (figure 2 A). During Phase II, experimental animals $(\mathrm{n}=12)$ were removed from immobilization bandages $3 x /$ week beginning at day 14 post-injury (instead of day 42) to perform free mobilization for 60 minutes $(n=4)$, treadmill walking for 30 minutes at $12 \mathrm{~m} / \mathrm{min}$ (instead of $10 \mathrm{~m} / \mathrm{min}$ ) plus free mobilization for 30 minutes $(n=4)$, or treadmill walking for 60 minutes at $12 \mathrm{~m} / \mathrm{min}(\mathrm{n}=4)$. Following their prescribed therapy each day (total of 60 minutes), animals were placed back in immobilization bandages. Because animals did not have several days of free mobilization before beginning treadmill walking as they did in Phase I, an acclimation period was designed in the walking protocol. Treadmill duration and speed were gradually increased during the first week until reaching the full 30 or 60 minutes at $12 \mathrm{~m} / \mathrm{min}$ on day 21 ; during this week of reduced treadmill time, animals spent the remaining time with free mobi- lization. Therapy continued until day 42 when bandages were permanently removed allowing free mobilization for an additional 42 days (i.e., until study day 84). One animal in the group performing treadmill walking for 60 minutes refused to walk so was removed from the study leaving only three animals in this group.

After reviewing results from Phase II, Phase III was designed to more aggressively investigate therapy intensity and duration (figure 2 B). During Phase III, experimental animals (n =9) began therapy at day 3 post-injury. This day was chosen because previous results with this animal model suggested the joint is likely unstable during these first few days (19). Limb use during this period could potentially cause damaging effects. One group of animals were removed from immobilization bandages $5 \mathrm{x} /$ week to perform treadmill walking for 30 minutes at $12 \mathrm{~m} / \mathrm{min}(\mathrm{n}=3)$. Following each therapy session, these animals were placed back in immobilization bandages similar to Phase II. Two separate groups were removed from immobilization bandages permanently at day 3 post-injury: one group performed only free mobilization $(n=3)$ while the other group performed treadmill walking for 30 minutes at $12 \mathrm{~m} / \mathrm{min} 5 \mathrm{x} /$ week in addition to the free mobilization $(\mathrm{n}=$ 3). Treadmill duration and speed were gradually increased during the first two sessions (day 3-4) until reaching the full 30 minutes at $12 \mathrm{~m} / \mathrm{min}$ on day 7; during these days of reduced treadmill time, animals spent the remaining time with free mobilization. Other ongoing studies determined that rangeof-motion (ROM) loss in this injury model was significant as early as day 21 post-injury (19). Therefore, Phase III was designed with a day 21 terminal time point including a separate control $(n=3)$ and injury $(n=3)$ group in order to significantly decrease the overall length of the animal study. Animals

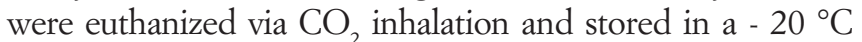
freezer at either day 84 (Phases I and II) or day 21 (Phase III).
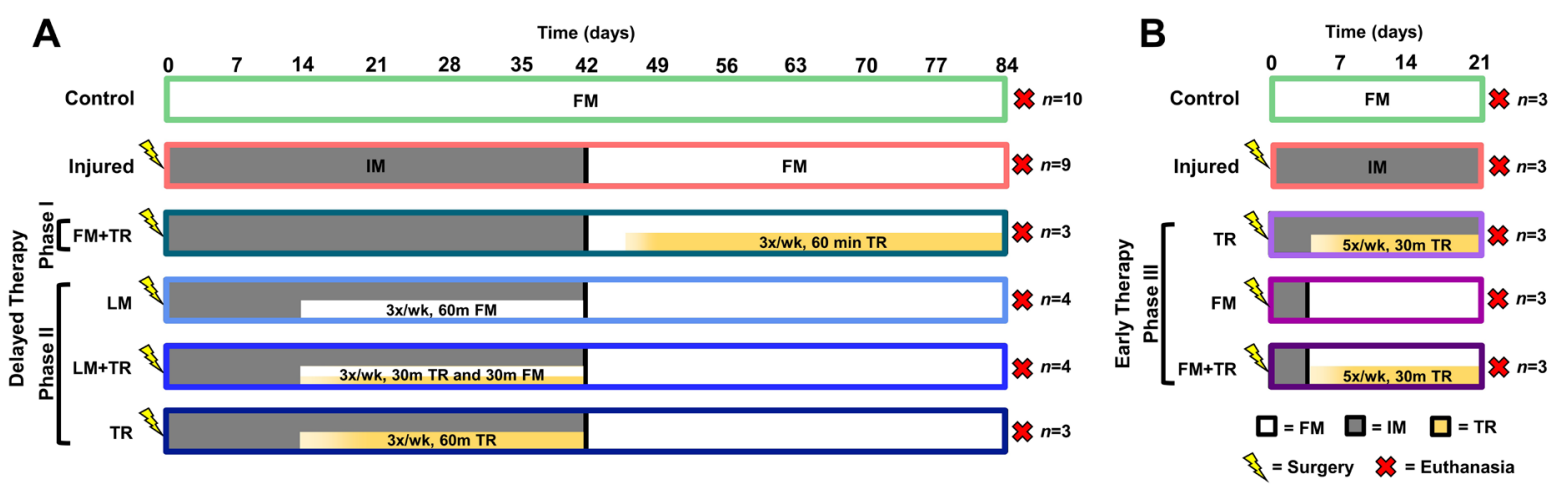

Figure 2. (A) Phase I and II delayed therapy and (B) Phase III early therapy protocols explored the timing, intensity and duration of active, functional exercise on joint motion outcomes.

IM: immobilization; FM: free mobilization; LM: time-limited free mobilization; TR: treadmill walking; group border color corresponds to data column shading in figure 4. 


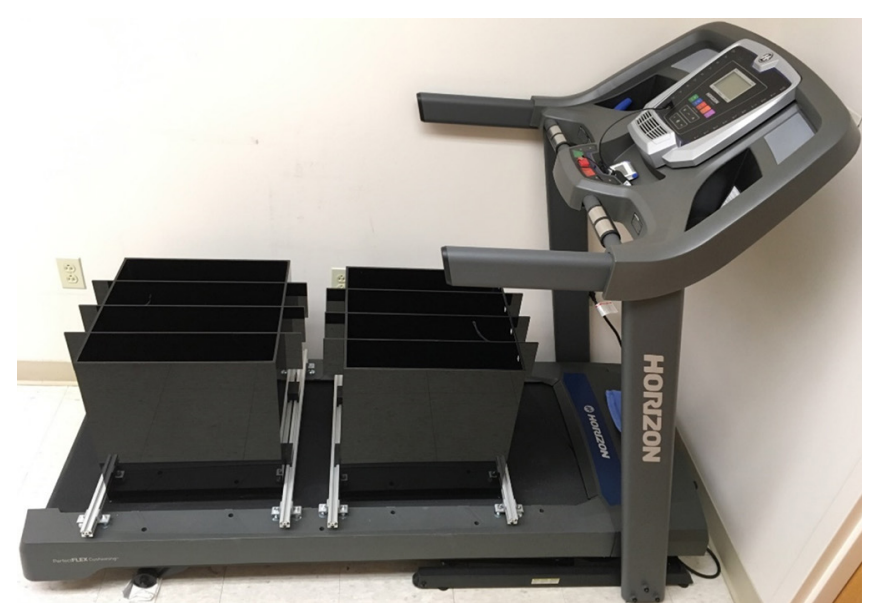

Figure 3. A home fitness treadmill with walking lane boxes mounted above was modified to reduce the speed range suitable for walking animals.

\section{Joint mechanical testing}

After thawing animals for 24 hours, both forelimbs from control animals (day 84: $\mathrm{n}=20$; day 21: $\mathrm{n}=6$ ) and injured forelimbs from injured (day 84: $\mathrm{n}=9$; day $21: \mathrm{n}=3$ ) and therapy ( $\mathrm{n}=3-4$ /group) animals were tested for flexion-extension joint motion in a custom mechanical system (17). Following an established testing protocol (17), limbs were loaded for five cycles to $\pm 11.25 \mathrm{~N} \times \mathrm{mm}$ at $1.0 \mathrm{~mm} / \mathrm{s}$ with overhead images taken at maximum flexion and extension. Torque and angular position data were analyzed using a MATLAB program (MathWorks, Natick, MA, USA) to quantify measurements of maximum flexion, maximum extension, total ROM and neutral zone (NZ) length (17). The NZ length describes the relatively resistance-free ROM represented by the flatter region on the torque-angular position curve between linear fits of the flexion and extension stiffness.

\section{Statistical analysis}

Within each Phase (i.e., I, II, or III), one-way analysis of variance (ANOVA) tests was used to compare each mechanical testing parameter between all groups. When significance was found, post-hoc comparisons with Bonferroni corrections compared the injured and experimental group(s) to the control group. Significance was defined as $\mathrm{p}<0.05$ for all tests.

\section{RESULTS}

The Phase I experimental group (delayed therapy beginning at day 42 post-injury) had a significantly larger maximum extension (figure $4 \mathrm{~A}, \mathrm{p}<0.0001$ ), smaller total ROM (figure $4 \mathrm{E}, \mathrm{p}<0.0001$ ) and smaller NZ length (figure $4 \mathbf{G}$, $\mathrm{p}<0.0001)$ compared to controls with values remaining similar in magnitude to injured animals (table I). Likewise, Phase II experimental groups (delayed therapy beginning at day 14 post-injury) exhibited significantly larger maximum extension (figure $4 \mathrm{~A}, \mathrm{p}<0.0001$ ), smaller total ROM (figure $4 \mathrm{E}, \mathrm{p} \leq 0.0002$ ) and smaller NZ length (figure $4 \mathrm{G}, \mathrm{p}$ $\leq 0.0002$ ) compared to controls with values remaining similar in magnitude to injured animals regardless of therapy intensity (table I). The Phase III experimental group (early therapy beginning at day 3 post-injury) performing treadmill walking but remaining in immobilization bandages after each session (Phase III TR) also showed no improvements; again, maximum extension (figure $4 \mathrm{~B}, \mathrm{p}<0.0001$ ), total ROM (figure $4 \mathrm{~F}, \mathrm{p}<0.0001$ ) and NZ length (figure $4 \mathrm{H}, \mathrm{p}$ $<0.0001$ ) were significantly different compared to controls with values remaining similar in magnitude to injured animals (table I). However, both Phase III experimental groups where limbs were permanently removed from immobilization bandages at day 3 post-injury (without and with additional treadmill walking, FM and FM + TR, respectively) showed large improvements. Maximum extension (figure $4 \mathrm{~B}$ ), total ROM (figure $4 \mathrm{~F}$ ) and NZ length (figure $4 \mathrm{H}$ ) in both therapy groups were only slightly, non-significantly different from controls $(\mathrm{p} \geq 0.7011)$ with differences $<10^{\circ}$ (table I). Comparatively, injured animals showed differences relative to controls in maximum extension, total ROM and NZ length of $61^{\circ}, 54^{\circ}$ and $45^{\circ}$, respectively (table I). Maximum flexion (figure $4 \mathrm{C}, \mathrm{D}$, table I) showed no differences across all groups. In this animal model, injury is not induced on the posterior joint and the limb is immobilized in flexion so changes in maximum flexion are not expected.

\section{DISCUSSION}

This work summarizes three chronological studies investigating the effects of early versus delayed therapy (timing), free mobilization versus forced treadmill walking (intensity), and limited-time versus unlimited use (duration) on joint motion outcomes. Previous work with this animal model concluded that following injury and 42 days of immobilization, joint motion was only partially restored with free mobilization from day 42 to 84 meaning that contracture persisted long-term $(18,19)$. Phase I increased the intensity of therapy via treadmill walking during this free mobilization period in an attempt to restore additional motion. Contrary to our hypothesis, joint motion outcomes showed that this protocol was ineffective (figure 4 A, E, G). As such, Phase II was developed in order to begin therapy earlier and vary intensity while maintaining the established 42-day immobilization protocol. Time-limited free mobilization or treadmill walking of injured limbs (with limbs remaining immobilized when not 


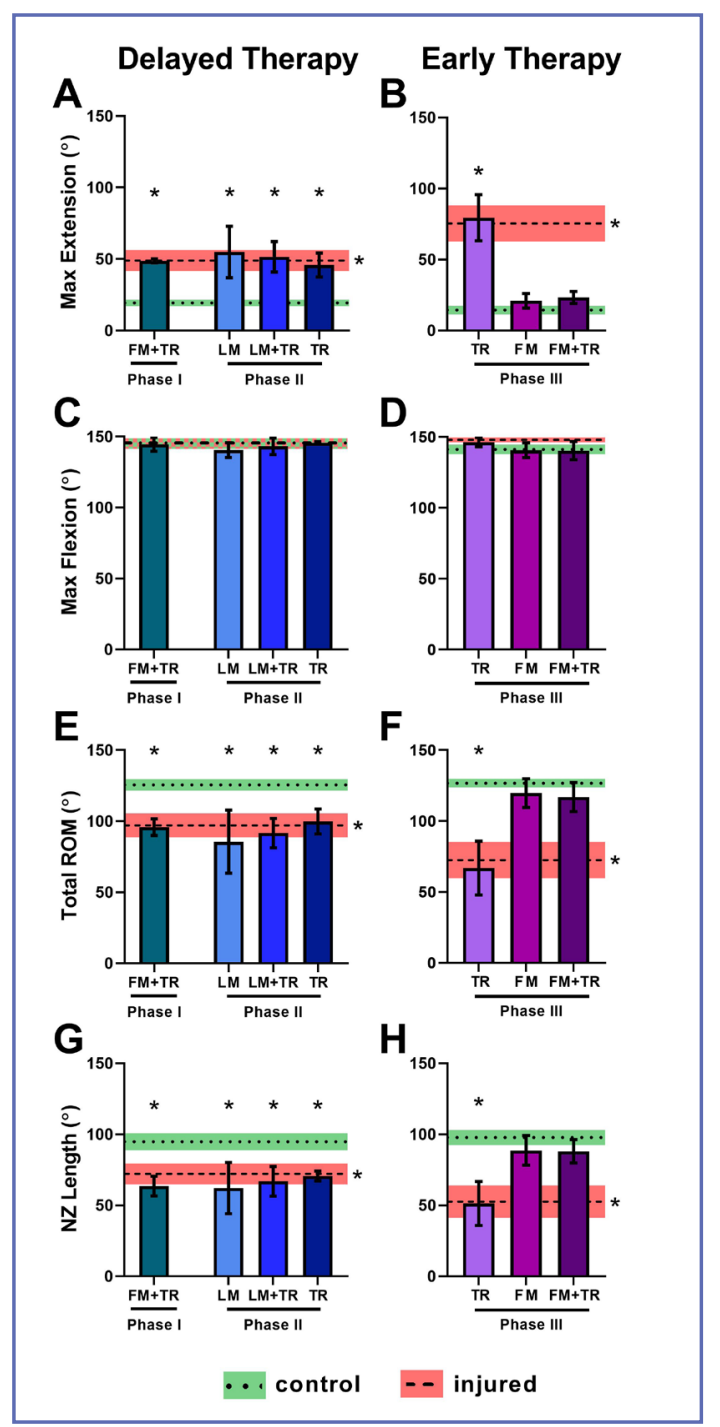

Figure 4. Phase I and Phase 2 experimental group had a significantly different (A) maximum extension, (E) total ROM and (G) NZ length compared to controls and remained similar to injured animals. Phase III experimental group performing treadmill walking but remaining in immobilization bandages after each session also showed no improvements having a significantly different (B) maximum extension, $(F)$ total ROM and $(\mathrm{H})$ NZ length compared to controls and remained similar to injured animals. However, both Phase III experimental groups that were permanently removed from immobilization bandages at day 3 post-injury showed large improvements in (B) maximum extension, (F) total ROM and (H) NZ length compared to injured limbs and were only slightly, non-significantly different from controls. As expected, (C, D) maximum flexion showed no differences across all groups.

ROM: range-of-motion; NZ: neutral zone; FM: free mobilization; LM: time-limited free mobilization; TR: treadmill walking; *: different from control; $p<0.05$; data column shading corresponds to group border color in figure 2 . conducting therapy) were expected to improve joint motion outcomes while allowing for proper healing to occur. Again, outcomes showed no improvements for any of the groups at day 84 (figure 4 A, E, G), leading to development/implementation of an aggressive Phase III early therapy protocol. Interestingly, treadmill walking 3 days post-injury $5 x$ /week (with limbs remaining immobilized when not conducting therapy) was still ineffective when compared to injured limbs receiving no therapy (figure $4 \mathrm{~B}, \mathrm{~F}, \mathrm{H}$ ). Only after removing bandages permanently at day 3 post-injury was there an improvement in biomechanical outcome, regardless of whether additional treadmill walking was performed (figure $4 \mathrm{~B}, \mathrm{~F}, \mathrm{H}$ ). Taken together, these results suggest that only early, unlimited use of the injured limbs have the potential to improve joint motion in this animal model of post-traumatic joint contracture.

Kaneguchi et al. saw no differences in rat knee ROM between animals receiving free mobilization with and without additional treadmill walking when knees were immobilized for 21 days (23). In our model, after 42 days of full immobilization (Phase I) or partial immobilization with time-limited limb use beginning day 14 post-injury (Phase II), altering intensity via treadmill walking showed no improvements similar to Kaneguchi et al. Trudel et al. concluded that immobilization effects were only fully reversible with free mobilization alone when immobilization was applied for 14 days or less (24). This result agrees with the Phase III data where joint ROM in injured animals was restored to $92 \%$ of control animal values when immobilization bandages were removed at day 3 post-injury. Sakakima et al. showed rat ankle ROM increased with free mobilization and treadmill walking when compared to free mobilization alone after 14 days of immobilization (21). It is possible that an immobilization length between 42 days (Phase I) and 3 days (Phase III) could show similar results. These previous results suggest that only minimal immobilization (i.e., less than 14 days) can prevent joint contracture and that treadmill walking has the potential to further increase $\mathrm{ROM}$ when free mobilization alone fails to do so. Importantly, none of these prior studies included a joint injury in addition to immobilization, as is the case in our rat elbow model. The presence of an acute, traumatic injury must be considered when developing therapy protocols. Joint instability and the possibility of causing secondary injuries is a serious concern with beginning therapy too early or aggressively. While beginning limb use day 3 post-injury might seem ideal for preventing contracture, early motion with a possible unstable joint could lead to cartilage and/or secondary soft tissue damage. These topics will be examined more closely in the future.

The competing ideas of early joint mobilization to benefit joint motion but joint immobilization to protect joint tissues from instability/further damage presents a dichotomy for 
Table I. Joint Motion Mechanical Testing Data.

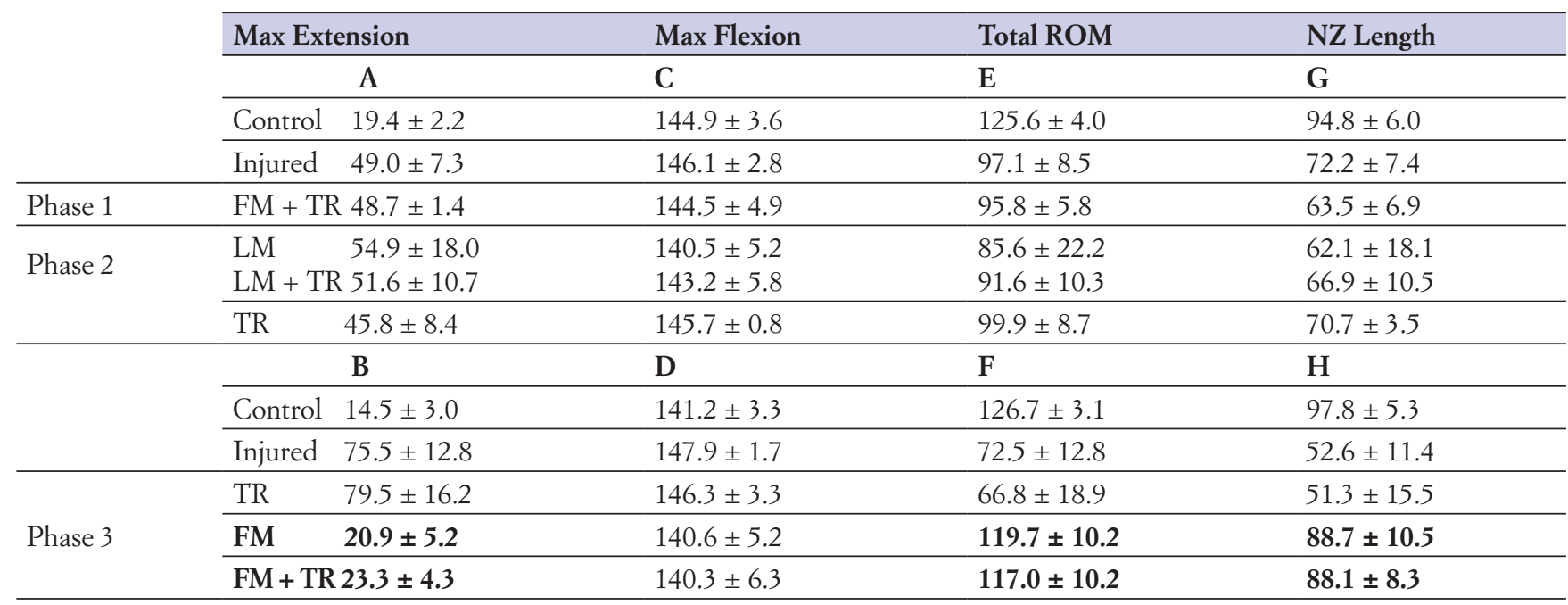

ROM: range-of-motion; NZ: neutral zone; FM: free mobilization; LM: time-limited free mobilization; TR: treadmill walking; column letter A-H corresponds to panel in figure 3; bolded values denotes successful therapy groups.

clinicians. Should a clinician focus on preventing contracture by beginning mobilization early at the risk of causing further damage, or do they delay mobilization to protect the joint at the risk of developing a permanent contracture? After a simple elbow dislocation, a protective brace is often used to lock the elbow at $90^{\circ}$ of flexion for the first $1-3$ weeks to limit extension and to avoid varus stress on the lateral collateral ligament (8). However, early mobilization after simple elbow dislocation resulted in an expedited return of ROM, function and return to work (25). Importantly, in either scenario, the severity of the damaged structures, motivation of the patient, and pain play a major role in the strategy a clinician chooses. Recently, Zhang et al. identified a lack of evidence quantifying the effects of the timing of active mobilization on final elbow ROM and function after trauma (6). Future work with our unique animal elbow model could help answer this question. By controlling for injury severity, means of active, functional joint exercises and therapy timing, intensity, and duration, results could help guide clinical practice on the appropriate timing of active mobilization and the risk of causing further damage in the context of simple elbow dislocation.

This study is not without limitations. A power analysis of previous data (power $=0.8 ; \alpha=0.05$ ) determined that $n=7 /$ group was required for joint mechanical tests to detect ROM differences of $15^{\circ}$ with a standard deviation of $10^{\circ}(19)$. All experimental groups, day 21 control group, and day 21 injured group had only $n=3-4$ /group in this study. However, results showed obvious, large differences (or no differences) between experimental groups and controls that would likely be maintained with a correctly powered study, so additional animals were not added to round out the full complement in each group. This study also included only post-mortem, joint-level motion data. While this provides useful data to build upon, other key outcomes including in vivo joint function or tissue microanatomy would provide a more comprehensive assessment of the effects of the various experimental physical therapy strategies. Nevertheless, previous work demonstrated that poor post-mortem joint motion data was associated with functional deficits of grip strength and gait, as well as pathological joint capsule tissue via histology, which would be expected in the various experimental groups where therapy was not effective $(17-20,26)$. Lastly, it is possible that treadmill walking was not the optimal forelimb physical therapy in rats. Treadmill walking has been successfully used as a hindlimb joint contracture therapy in rodents $(21,23,27)$ but, to our knowledge, we are the first to use it for forelimb therapy. While walking, injured forelimbs were confirmed to be used for locomotion and not maintained in an elevated, flexed position where the animals only walked on the other three limbs. However, the actual ROM of the elbow during use could not be confirmed. It is currently unknown whether full or any partial extension of the elbow is necessary to see a beneficial effect. Future work could incorporate various elbow extension amounts via treadmill walking or other therapy means as well as the timing, duration or intensity in protocols.

\section{CONCLUSIONS}

In conclusion, results of this study showed that early motion is essential to preserving ROM following a traumatic elbow injury in a rat model of PTJC. To address these findings, 
ongoing work is investigating the effect of post-injury immobilization and free mobilization duration on joint motion outcomes. Additionally, studies will explore the potential for treadmill walking or other physical therapy activities to further increase joint motion when not completely restored with free mobilization alone. Furthermore, in vivo joint function and tissue histological analyses will be includ-

\section{REFERENCES}

1. Gaston P, Will EM, Keating JF. Recovery of knee function following fracture of the tibial plateau. J Bone Jt Surg $\mathrm{Br}$ 2005;87-B(9):1233-6.

2. Anakwe RE, Middleton SD, Jenkins PJ, McQueen MM, CourtBrown CM. Patient-Reported Outcomes After Simple Dislocation of the Elbow. J Bone Jt Surg Am 2011;93(13):1220-6.

3. Kuhn MA, Ross G. Acute Elbow Dislocations. Orthop Clin North Am 2008;39(2):155-61.

4. Charalambous CP, Morrey BF. Posttraumatic elbow stiffness. J Bone Jt Surg Am 2012;94(15):1428-37.

5. Monument MJ, Hart DA, Salo PT, Befus AD, Hildebrand KA. Posttraumatic elbow contractures: targeting neuroinflammatory fibrogenic mechanisms. J Orthop Sci 2013;18:869-77.

6. Zhang D, Nazarian A, Rodriguez EK. Post-traumatic elbow stiffness: Pathogenesis and current treatments. Shoulder Elb 2020;12(1):38-45.

7. Jones V. Conservative management of the post-traumatic stiff elbow: a physiotherapist's perspective. Shoulder Elb Elb 2016;8(2):134-41.

8. Marinelli A, Guerra E, Ritali A, Rotini R. Elbow Brace and Rehabilitation. In: Bain G, Eygendaal D, van Riet RP (eds). Surgical Techniques for Trauma and Sports Related Injuries of the Elbow. Berlin Heidelberg: Springer, 2020:p. 811-8.

9. Hackl M, Beyer F, Wegmann K, Leschinger T, Burkhart KJ, Müller LP. The treatment of simple elbow dislocation in adults. Dtsch Arztebl Int 2015;112(18):311-9.

10. Michlovitz SL, Harris BA, Watkins MP. Therapy interventions for improving joint range of motion: A systematic review. J Hand Ther 2004;17(2):118-31.

11. Myden C, Hildebrand K. Elbow joint contracture after traumatic injury. J Shoulder Elb Surg 2011;20(1):39-44.

12. Everding NG, Maschke SD, Hoyen HA, Evans PJ. Prevention and treatment of elbow stiffness: A 5 -year update. J Hand Surg Am 2013;38(12):2496-507.

13. Fusaro I, Orsini S, Stignani Kantar S, Sforza T, Benedetti MG, Bettelli $G$, et al. Elbow rehabilitation in traumatic pathology. Musculoskelet Surg 2014;98(Suppl 1):95-102.

14. Taylor F, Sims M, Theis J, Herbison G. Interventions for treating acute elbow dislocations in adults. Cochrane Database Syst Rev 2012;(4):CD007908.

15. Harding P, Rasekaba T, Smirneos L, Holland AE. Early mobilisation for elbow fractures in adults. Cochrane Database Syst Rev 2011;(6):CD008130.

16. MacDermid JC, Vincent JI, Kieffer L, Kieffer A, Demaiter J, MacIntosh S. A Survey of Practice Patterns for Rehabilitation Post Elbow Fracture. Open Orthop J 2012;6(1):429-39.

17. Lake SP, Castile RM, Borinsky S, Dunham CL, Havlioglu N, Galatz LM. Development and Use of an Animal Model ed to compliment post-mortem joint motion outcomes and address concerns of joint instability or secondary injuries associated with beginning therapy too early or aggressively.

\section{CONFLICT OF INTERESTS}

The authors declare that they have no conflict of interests (28).

to Study Post-Traumatic Stiffness and Contracture of the Elbow. J Orthop Res 2016;34(2):354-64.

18. Dunham CL, Castile RM, Havlioglu N, Chamberlain AM, Galatz LM, Lake SP. Persistent motion loss after free joint mobilization in a rat model of post-traumatic elbow contracture. J Shoulder Elb Surg 2017;26(4):611-8.

19. Dunham CL, Castile RM, Havlioglu N, Chamberlain AM, Lake SP. Temporal Patterns of Motion in Flexion-extension and Pronation-supination in a Rat Model of Posttraumatic Elbow Contracture. Clin Orthop Relat Res 2018;476(9):1878-89.

20. Reiter AJ, Kivitz GJ, Castile RM, et al. Functional Measures of Grip Strength and Gait Remain Altered Long-term in a Rat Model of Post-traumatic Elbow Contracture. J Biomech Eng 2019;141(7):071001.

21. Sakakima H, Yoshida Y, Sakae K, Morimoto N. Different frequency treadmill running in immobilization-induced muscle atrophy and ankle joint contracture of rats. Scand J Med Sci Sport 2004;14(3):186-92.

22. Rooney SI, Loro E, Sarver JJ, et al. Exercise protocol induces muscle, tendon, and bone adaptations in the rat shoulder. Muscles Ligaments Tendons J 2014;4(4):413-9.

23. Kaneguchi A, Ozawa J, Minamimoto K, Yamaoka K. Active exercise on immobilization-induced contractured rat knees develops arthrogenic joint contracture with pathological changes. J Appl Physiol 2018;124(2):291-301.

24. Trudel G, Laneuville O, Coletta E, Goudreau L, Uhthoff HK. Quantitative and temporal differential recovery of articular and muscular limitations of knee joint contractures; results in a rat model. J Appl Physiol 2014;117(7):730-7.

25. Catapano M, Pupic N, Multani I, Wasserstein D, Henry P. Early functional mobilization for non-operative treatment of simple elbow dislocations: a systematic review. Shoulder Elb 2020;0(0):1-11.

26. Reiter AJ, Schott HR, Castile RM, Cannon PC, Havlioglu $\mathrm{N}$, Chamberlain AM, et al. Females and males exhibit similar functional, mechanical, and morphological outcomes in a rat model of posttraumatic elbow contracture. J Orthop Res 2020;1-11.

27. Morimoto A, Winaga H, Sakurai H, Ohmichi M, Yoshimoto T, Ohmichi Y, et al. Treadmill running and static stretching improve long-lasting hyperalgesia, joint limitation, and muscle atrophy induced by cast immobilization in rats. Neurosci Lett 2013;534(1):295-300.

28. Padulo J, Oliva F, Frizziero A, Maffulli N. Basic principles and recommendations in clinical and field science research: 2018 update. Muscles Ligaments Tendons J 2018;8(3):305-7. 